\title{
Leçons tirées de l'accident de Goiânia, au Brésil
}

\author{
J.R. CROFT*
}

(Manuscrit reçu le 15 décembre 1989)

\begin{abstract}
RÉSUMÉ Les accidents d'irradiation hors du secteur nucléaire sont responsables d'une proportion non négligeable d'irradiations, fatales ou non. Après un court rappel des statistiques en ce domaine, l'article décrit l'un des accidents radiologiques les plus graves, celui de Goiânia, au Brésil, en 1987. L'accident entraîna le décès de quatre personnes et une forte dispersion de la contamination radioactive due à la rupture d'une source de radiothérapie. Sont relatés, en particulier, le déroulement de l'accident, les mesures prises, les leçons tirées.

ABSTRACT Radiation accidents outside of the nuclear sector account for a significant proportion of fatal and non-fatal radiation accidents. Relevant statistics are briefly reviewed and then the paper concentrates on one of the most serious radiological accidents, that in Goiânia, Brazil, in 1987. In that accident four people died and there was significant spread of radioactive contamination from a ruptured radiotherapy source. The development of the accident, the response to it and the lessons learned from it are addressed.
\end{abstract}

\section{AVANT-PROPOS}

Un nombre significatif d'accidents mortels ou non mortels par irradiation ne relève pas du domaine nucléaire. Après un bref rappel des statistiques, nous procédons à l'étude d'un des accidents radiologiques les plus graves, celui de Goiânia, au Brésil, survenu en 1987. Cet accident a causé la mort de quatre personnes et a provoqué une importante dispersion de la contamination radioactive par suite de la rupture de l'enveloppe de la source de radiothérapie. Nous retraçons le déroulement de l'accident et les interventions pour y remédier et les leçons qui en ont été tirées.

\section{INTRODUCTION}

Dès que l'on aborde le sujet des accidents par irradiation, nombreux sont ceux qui pensent immédiatement aux accidents des centrales nucléaires ou liés au cycle du combustible núcléaire. Ce n'est pas étonnant si l'on pense à tout ce qu'ont pu dire et écrire les hommes politiques et les médias. En fait, une forte proportion d'accidents est imputable au secteur non nucléaire ; elle est liée à l'utilisation de sources radioactives et d'équipements aux rayons $X$, dans les domaines de l'industrie, de la médecine ou de la recherche scientifique. On regroupe tous ces accidents sous la dénomination d'"accidents radiologiques".

* National radiological protection board, Chilton, Didcot, Oxon OX11 ORQ, GrandeBretagne. 
Après avoir rapidement passé en revue les statistiques correspondantes, nous nous attachons à l'étude d'un des plus graves accidents radiologiques qui se soit jamais produit, à savoir celui de Goiânia, au Brésil, survenu en 1987. Dans cet accident, l'enveloppe de la source radioactive de haute activité a été rompue et les substances radioactives se sont répandues dans la ville. De nombreuses personnes ont reçu de fortes doses de rayonnements soit par irradiation externe, soit par incorporation de substances radiologiques. Quatre personnes sont décédées et vingt-huit ont subi des brûlures radiologiques. L'importance et le degré de contamination de certains quartiers de la ville étaient tels qu'il a fallu démolir des constructions et enlever dans ces zones la couche de terre superficielle. Cette étude retrace le déroulement de l'accident, les interventions et les leçons que nous pouvons en tirer.

\section{STATISTIQUES EN MATIĖRE D'ACCIDENTS}

L'Agence internationale de l'énergie atomique (AIEA) a publié les statistiques sur les accidents mortels signalés entre 1945-1987 (tableau I) [1]. (Elles ne tiennent pas compte de deux cas de suicide avec des sources scellées et de trois décès imputables à l'effet de souffle lors de l'accident du réacteur de recherche SL1, survenu en 1961). On peut ajouter, dans la catégorie des accidents radiologiques, un autre décès récent consécutif à un accident dans une installation au Salvador [2] destinée à la stérilisation d'instruments médicaux par irradiation.

TABLEAU 1

Accidents mortels, 1945-1987

\begin{tabular}{|c|c|c|c|}
\hline \multicolumn{2}{|c|}{ Trpe dPaccident } & Wombre dPaccidents & Worts \\
\hline Nucléaire & $\begin{array}{l}\text { - Tchernobyl } \\
\text { - autres nucléaires }\end{array}$ & $\begin{array}{l}1 \\
6\end{array}$ & $\begin{array}{r}29 \\
6\end{array}$ \\
\hline Radiologique & $\begin{array}{l}\text { - radiographie gamma } \\
\text { - autres }\end{array}$ & $\begin{array}{l}4 \\
6\end{array}$ & $\begin{array}{l}14 \\
10\end{array}$ \\
\hline
\end{tabular}

S'il est vrai que les accidents nucléaires, et notamment celui de Tchernobyl, sont des accidents très graves, il n'en est pas moins vrai que les accidents non nucléaires ou "radiologiques" sont responsables d'un nombre important de morts et méritent à ce titre qu'on s'y intéresse. Si l'on voulait étendre les statistiques aux accidents non mortels, les résultats seraient sensiblement les mêmes. Pour la période de 1945 à 1985, Oliveira a enregistré [7] 178 accidents impliquant 324 personnes exposées de façon significative. Au début de cette période, ce sont les accidents de type criticité qui ont dominé, puis ils ont considérablement décru au cours des dernières décades, à l'exception, toutefois, de Tchernobyl. La majeure partie des accidents - 153 impliquant 255 personnes - sont de type radiologique, liés principalement à la gammagraphie 
industrielle, à la cristallographie aux rayons $X$, à la radiographie aux rayons $X$ pour l'industrie et la recherche, aux accélérateurs de recherche, à la radiothérapie et aux installations de stérilisation / irradiation. La gammagraphie, à elle seule, totalise plus de $40 \%$ des accidents radiologiques. Ceux-ci sont essentiellement imputables d'une part à une formation insuffisante et d'autre part à des procédés inadéquats notamment en matière de sécurité de la source. Dans certains cas, cela s'est même terminé par le contact des sources avec le public, entraînant des morts, comme à Mexico en 1962 [4], en Algérie en 1978 [1] et au Maroc en 1984 [6].

Les accidents avec contamination du public sont, jusqu'à présent, peu nombreux et peu importants. L'intervention en cas d'accident en dehors du secteur nucléaire a été, dans l'ensemble, organisée pour traiter de sources perdues avec des problèmes de contamination très limitée. Ces dernières années, on a constaté que, dans des accidents étonnamment similaires comme celui de Juarez au Mexique (1983) [6] et de Goiânia au Brésil (1987), toutes les conditions étaient réunies pour qu'une dispersion à grande échelle de substances radioactives se produise dans le domaine public. Nous résumons ci-dessous les principaux points du rapport réalisé par l'AIEA sur l'accident de Goiânia [3].

\section{L'ACCIDENT DE GOIANIA}

\section{Historique de l'accident}

Goiânia est une grande ville peuplée d'environ un million d'habitants. Elle est la capitale de l'Etat de Goias, situé sur le plateau central brésilien. Elle est à environ $1000 \mathrm{~km}$ des villes de Rio de Janeiro et de São Paulo où se trouvent les principaux équipements du pays en matière de radioprotection. L'Instituto Goiano de Radioterapia (IGR), à Goiânia, était une clinique privée de radiothérapie, appartenant à une association de médecins, qui a commencé son activité en juin 1971. Les locaux de la clinique comprenaient des salles de traitement où l'on utilisait des unités de téléthérapie au césium 137 et au cobalt 60 , agréées par les autorités compétentes brésiliennes, à savoir la Commission nationale d'énergie nucléaire (CNEN).

Vers la fin de l'année 1985, l'IGR a cessé d'utiliser ces locaux et une nouvelle association de médecins a repris l'activité de la clinique en la transférant ailleurs. L'unité de cobalt 60 a été déménagée dans les nouveaux locaux mais, comme à l'occasion de la dissolution de la société, la propriété de l'équipement de l'ancienne clinique faisait l'objet d'un litige, l'unité au césium est restée sur place.

La CNEN n'en a pas été informée et, dans les mois qui ont suivi, une grande partie de la clinique et de ses annexes a été démolie avec la perspective d'un réaménagement du site. Les salles de traitement n'ont pas été démolies et sont restées à l'abandon servant, semble-t-il, à des 
vagabonds. Deux années se sont écoulées péndant lesquelles l'unité de téléthérapie est demeurée dans cette: situation très peu sûre.

L'unité de téléthérapie renfermait une source de césium 137 avec une activité de $50,9 \mathrm{TBq}(1375 \mathrm{Ci})$. La source était située dans un barillet à l'intérieur d'une enveloppe blindée en plomb et acier, si bien qu'en tournant on pouvait mettre la source sur la position "exposition" ou "sécurité". La substance radioactive se présentait sous forme de chlorure de césium très soluble, rendu compact de manière à former une masse cohérente, conditionné dans une deuxième enveloppe en acier inoxydable et placé dans une "capsule internationale" de dimensions normalisées. Bien qu'actuellement les sources au césium soient presque toujours sous forme vitrifiée non dispersable, on a utilisé, pour les sources de téléthérapie, le césium sous la forme moins souhaitable de chlorure de césium car la forme vitrifiée ne permet pas d'atteindre l'activité spécifique nécessaire.

\section{Enlèvement et rupture de la source}

Vers la mi-septembre de 1987, deux hommes, R.A. et W.P., entendent dire que du matériel de valeur a été laissé dans l'IGR. Ils se rendent sur le site abandonné et à l'aide d'outils rudimentaires tentent de démonter l'appareil de téléthérapie dans le but de le vendre à la ferraille, inconscients des dangers courus. Le 13 septembre, ils parviennent enfin à démonter le barillet porte-source et l'emportent sur une brouette chez R.A. On peut considérer, en un certain sens, que l'enveloppe de la source n'était plus intacte et que les deux hommes ont pu être exposés à des doses de 4,6 Gy $\mathrm{h}^{-1}$ à $1 \mathrm{~m}$. Le jour suivant, les deux hommes ont des vomissements et W.P., qui a de surcroît une main enflée, consulte un médecin. Celui-ci attribue les symptômes observés à une espèce de réaction allergique à ce que le patient a mangé. Le 18 septembre, les deux hommes s'attaquent de nouveau au barillet dans le jardin de R.A., tapent avec un marteau sur un tournevis pour percer la "fenêtre" en acier de l'enveloppe de la source, de $1 \mathrm{~mm}$ d'épaisseur, et ils y parviennent. Lorsqu'on a mesuré, ultérieurement, le débit de dose à proximité de cet endroit, la contamination résiduelle était de 1,1 Gy $h^{-1}$. Cependant, comme les deux hommes ont l'impression qu'ils ne pourront pas obtenir ce qu'ils considèrent comme des métaux de valeur, ils vendent le barillet à un premier ferrailleur, D.F. Ce soir-là, en entrant dans son garage où était placé le barillet, l'homme voit une espèce de lueur bleue qui en émane. Fasciné, pensant qu'il s'agit de quelque chose de valeur ou peutêtre même de surnaturel, il l'emporte chez lui pour le montrer à sa femme M.F.1. Les jours suivants, de nombreux amis et parents viennent voir ce phénomène; certains sont même autorisés à extraire des fragments de la source, de la taille d'un grain de riz, qui peuvent facilement être réduits en poudre. L'exemple peut-être le plus tragique de cette distribution est celui de la famille d'I.F., frère de D.F., qui emporte chez lui des fragments. Au cours du repas, I.F. dépose les fragments sur la table. Sa fille de 6 ans, L.F.2, les manie tout en mangeant avec les mains. La petite n'a pas survécu ; on estime qu'elle $a$ incorporé $1,0 \mathrm{GBq}$ et reçu une dose 
estimée à 6,0 Gy. Après la distribution des fragments de la source, le barillet a été découpé par deux ouvriers de D.F., I.S. et A.S., qui sont également décédés, ayant reçu respectivement des doses estimées à 4,5 et 5,3 Gy. Les différentes pièces de la source ont ensuite été distribuées à deux autres ferrailleurs.

Pendant la période du 13 au 28 septembre, la radioactivité s'est largement dispersée par différents moyens. Au début, elle a été transportée en faible quantité par des personnes et des objets contaminés. Cependant, sa forme hautement soluble liée aux conditions climatiques ont rendu cette contamination très mobile.

On a signalé un certain nombre de cas où les personnes ont mis de cette poudre sur la peau parce qu'elle ressemblait à une substance scintillante utilisée pour le Mardi Gras. II ne fait aucun doute que la forme chimique de la source et le phénomène de lueur bleutée ont eu un profond effet sur le déroulement de l'accident. Contrairement à la croyance populaire, une substance radioactive ne produit normalement pas de lueur dans le noir. Ce phénomène n'avait pas encore été observé par les fabricants de sources ; par la suite, cette lueur bleue a, en revanche, été observée par le Laboratoire national d'Oak Ridge, aux USA, en enlevant l'enveloppe d'une source similaire. On s'est demandé si elle n'était pas associée à la fluorescence ou à un effet Cerenkov dû à l'absorption d'humidité.

\section{Découverte}

A la date du 28 septembre, un nombre non négligeable de personnes sont malades dont 10 en traitement à l'hôpital des maladies tropicales. M.F.1, la femme du ferrailleur, est intuitivement convaincue que la poudre bleue en est responsable. Avec l'un des ouvriers de son mari, G.S., elle se rend à la Vigilancia sanitaria emportant le restant de la source dans un sac que l'ouvrier porte sur ses épaules. Elle explique à la Vigilancia sanitaria (mi-cabinet médical, mi-centre d'hygiène) que cet objet est en train de "tuer sa famille". Malade elle-même, elle est hospitalisée et meurt ayant reçu une dose estimée à 4,3 Gy. Le médecin, ignorant ce que peuvent être ces restes de source, les dépose dans la cour pendant qu'il mène son enquête. Dans le même temps, les médecins sont de plus en plus inquiets sur le sort de leurs patients de l'hôpital des maladies tropicales, ne parvenant pas à déterminer la cause de la maladie. Parmi les causes évoquées, ils pensent à une intoxication alimentaire, à des dermites de contact et à un pemphigus. Toutefois, un médecin commence à soupçonner que les lésions cutanées pourraient être imputables à des radiations. Ses investigations auprès de confrères se recoupent avec celles de la Vigilancia sanitaria. Le lendemain, 29 septembre, on demande à un spécialiste de physique médicale en visite dans la ville, d'examiner le paquet avec un débitmètre emprunté. II est effrayé par les résultats : le paquet renferme encore 4,5 TBq. Une visite au "ferrailleur 1 " révèle des zones de contamination importante. De sa propre initiative, le physicien fait évacuer la Vigilancia sanitaria et la maison du "ferrailleur 1" ainsi que ses environs. 


\section{Premières actions}

La situation est signalée aux autorités gouvernementales de Goiânia. Comme on peut aisément l'imaginer, celles-ci ne veulent pas croire à l'ampleur potentielle de l'accident. II faut beaucoup de persévérance au physicien et ce n'est qu'au bout de plusieurs heures qu'il arrive à voir le secrétaire d'Etat à la santé qu'il informe de la situation. Un coordonnateur des situations d'urgence (NEC) de la Commission nationale d'énergie nucléaire (CNEN) à Rio de Janeiro est avisé. Celui-ci ordonne la mobilisation du personnel spécialisé, puis part pour Goiânia afin de se rendre compte de la situation. A ce stade, alors que la situation est jugée sérieuse, l'ampleur et les origines de l'incident ne sont pas encore connues.

\section{Action locale}

Les autorités de Goiânia mobilisent la police, les pompiers et la sécurité civile et décident que le stade olympique, situé à proximité, sera le point de rassemblement et de contrôle. Entre-temps, le physicien avec la collaboration de la nouvelle clinique IGR continue de contrôler autour des zones de contamination connues et de rechercher de nouvelles zones, sur la base du récit des événements qui lui est fait par les habitants. La sélection initiale des personnes envoyées au stade olympique est essentiellement faite en fonction du contact probable avec des parties de la source, c'est-à-dire la famille et les voisins des principaux protagonistes.

Bien qu'il n'existe pas de plan d'intervention pour répondre à cette situation d'urgence, la stratégie improvisée par les autorités a permis de maîtriser la situation et de prévenir d'autres expositions sérieuses. Dès l'arrivée du personnel de la CNEN, les autorités locales ont commencé à leur laisser toute responsabilité en matière de contrôle tout en continuant à apporter leur concours.

\section{Action de la CNEN}

Etant donné les distances, l'expert NEC et ses deux assistants techniques ne sont arrivés qu'après minuit. L'expert NEC s'est rendu rapidement compte de l'ampleur de l'accident, bien plus grave qu'on ne l'avait cru à l'origine. II a donc demandé d'autres secours. Le jour suivant, le $1^{\text {er }}$ octobre, on compte environ vingt experts et quatre principales zones de contamination sont isolées (maison de R.A. et trois chantiers de ferrailles). Au cours des deux journées suivantes, trois foyers supplémentaires et d'autres zones de contamination sont identifiées et isolées. Quelque 200 personnes sont évacuées de 41 maisons. Certaines avaient été évacuées par le physicien sur la base du niveau d'action en situation normale et non selon les niveaux prévus en cas d'urgence. L'équipe de contrôle du stade olympique identifie 249 personnes porteuses d'une contamination détectable, dont 121 avec contamination interne. Le 3 octobre, la phase initiale de prise de contrôle est achevée. 


\section{Conséquences humaines}

A leur arrivée à Goiânia, les médecins spécialisés en radioprotection sont confrontés au cas des 11 patients déjà hospitalisés à l'hôpital des maladies tropicales, auxquels s'ajoutent 22 personnes rassemblées au stade olympique chez qui on a décelé une contamination externe et interne.

Cependant, après un tri initial et une décontamination externe, on estime que le problème le plus urgent est celui des patients de l'hôpital des maladies tropicales qui souffrent toutes du syndrome d'irradiation aigu ou de lésions cutanées. Ces patients sont transférés dans une aile évacuée de l'hôpital général de Goiânia ; on procède au contrôle de la contamination avec prélèvement d'échantillons de sang, d'urine et de selles. Les semaines suivantes d'autres patients sont admis. Entre le $1^{\text {er }}$ et le 3 octobre, dix patients jugés gravement atteints sont transférés par avion à l'hôpital naval Marcilio Dias à Rio, prévu pour recevoir les victimes d'accident d'irradiation aux termes du plan d'intervention pour la centrale nucléaire d'Angra située au sud de Rio de Janeiro. La communauté médicale de Goiânia, le personnel de laboratoire et celui de l'hôpital naval ont montré quelques réticences à collaborer, craignant pour leur santé. II a fallu surmonter ce problème en demandant aux spécialistes de rassurer tout le monde et de donner des instructions informelles.

Pour les patients les plus gravement irradiés, le traitement a consisté à évaluer la crise hématologique associée au syndrôme d'irradiation aiguë. En dehors des symptômes cliniques, les médecins se sont efforcés d'estimer l'ordre de grandeur des doses par les techniques cytogénétiques d'analyse d'aberrations chromosomiques (tableau II). Pour l'évaluation de la contamination interne, on a d'abord eu recours aux analyses radiotoxicologiques à partir de la mesure de la radioactivité dans les urines et les fèces. A la mi-novembre, des installations temporaires d'anthroporadiamétrie ont permis de mesurer directement la contamination interne des patients et de confirmer les résultats des analyses biologiques.

Quatre victimes sont mortes dans les quatre semaines qui ont suivi leur admission à l'hôpital. Sur les vingt patients hospitalisés, dix-neuf présentaient des brûlures radio-induites. Toütes les brûlures étaient contaminées et les débits de dose pouvaient atteindre $15 \mathrm{mSv} \mathrm{h}^{-1}$ près de la peau. Le contrôle de la contamination était un problème majeur étant donné la sécrétion continuelle du césium 137 par la sueur qui faisait des patients une source de contamination mobile et régénérablé.

Les niveaux de contamination interne dus au césium 137, provoqués par l'accident de Goiânia, sont les plus élevés qui aient jamais été enregistrés : $1 \mathrm{GBq}$ pour une fillette de 6 ans décédée par la suite. La contamination interne a affecté 121 personnes et l'accélération de la décorporation a été un problème majeur. Pour 62 personnes, leur charge corporelle a requis l'utilisation du bleu de Prusse (hexacyanoferrate de potassium) recommandé dans de telles situations. Son efficacité a été démontrée, mais il a fallu augmenter les doses recommandées jusqu'alors. 


\section{TABLEAU II}

Estimation cytogénétique initiale des doses (en Gy)

\begin{tabular}{|lllll|}
\hline DF & 7,0 & propriétaire de l'entrepôt de ferraille $n^{\circ} 1$ & \\
\hline \hline RA & 6,2 & a pris la source & Décédée \\
\hline LF2 & 6,0 & nièce de DF & Décédée \\
\hline \hline MF1 & 5,7 & épouse de DF & Décédé \\
\hline AS & 5,3 & ouvrier de DF & Décédé \\
\hline IS & 4,5 & a travaillé sur la source & \\
\hline \hline EF2 & 4,4 & voisin de DF & \\
\hline MA1 & 4,3 & belle-mère de DF & \\
\hline LM & 4,0 & $\begin{array}{l}\text { épouse de l'ouvrier de l'entrepôt } \\
\text { de ferraille } n^{\circ} 2\end{array}$ & \\
\hline GS & 3,0 & $\begin{array}{l}\text { ouvrier de DF, s'est rendu à la } \\
\text { Vigilancia sanitaria }\end{array}$ \\
\hline \hline IF & 3,0 & $\begin{array}{l}\text { frère de DF, propriétaire de l'entrepôt } \\
\text { de ferraille } n^{\circ} 2\end{array}$ \\
\hline WP & 2,7 & \begin{tabular}{l} 
a pris la source \\
\hline 9
\end{tabular} \\
\hline
\end{tabular}

\section{Conséquences physiques}

A la date du 3 octobre, les sept principaux foyers de contamination étaient entièrement identifiés et maîtrisés. Pour être sûr qu'aucune autre zone majeure de contamination n'avait été oubliée, on a effectué, peu après, un relevé aérien à l'aide d'un hélicoptère spécialement équipé. On s'est aperçu des limites de cette technique, surtout à proximité des principaux foyers; on a donc eu recours à un équipement de surveillance installé sur une voiture. Parallèlement à ces techniques, on a demandé aux habitants des lieux contaminés d'indiquer leurs déplacements durant cette même période ainsi que ceux de leurs visiteurs. Sur la base de ces éléments, on a tracé sur une carte les voies potentielles de transport de la contamination ; 42 autres sites moins touchés ont ainsi été découverts.

Très tôt, une décision stratégique avait été rapidement prise : aucune décontamination majeure ne serait entreprise tant que des relevés complets ne seraient pas effectués et qu'un plan cohérent de travail ne serait pas dressé. Ces relevés ont été effectués à pied à l'aide de détecteurs à main. En raison des fortes pluies tombées sur la ville pendant l'accident, on avait d'abord pensé que la radioactivité serait entraînée dans le sol 
et retenue. Les relevés ont montré qu'il n'en était rien. II semble que les températures élevées (atteignant $40^{\circ} \mathrm{C}$ ) aient rapidement assécher le sol et que des vents forts aient provoqué une remise en suspension. L'ampleur des effets a véritablement surpris ; par exemple, le débit de dose à l'intérieur des habitations était dû essentiellement à la contamination déposée sur le toit des constructions à un étage. Parallèlement à ces relevés, on a entrepris un programme extensif de contrôle de l'environnement par prélèvement d'échantillons de sol, de végétaux, d'eau et d'air. Des contre-mesures n'ont été nécessaires que pour le sol et les fruits, dans la limite d'un rayon de $50 \mathrm{~m}$ autour des principaux foyers.

Pendant la phase de réhabilitation, il n'a pas été facile de réunir à $1000 \mathrm{~km}$ des principaux centres d'examens radiologiques la totalité du personnel et de l'équipement nécessaires. On a estimé au début que le programme de décontamination concernerait 4 à $5000 \mathrm{~m}^{3}$ de déchets radioactifs (il y en a eu, en fait, $3500 \mathrm{~m}^{3}$ ). Décider du type d'emballage, du site de stockage des déchets, de sa conception et de sa réalisation a demandé plus de temps que prévu. II fallut une décision politique pour choisir un site de stockage temporaire des déchets pour une période maximale de 2 ans. Ce n'est qu'à la mi-novembre qu'il a été possible de commencer les grands travaux de décontamination. Cela a abouti, entre autres, à la démolition de sept maisons, à l'enlèvement de la couche de terre superficielle et, pour certaines, le recouvrement du sol par du béton, à la décontamination de voitures, de maisons et de leur contenu. Cette phase s'est achevée avec succès le 21 décembre, permettant ainsi aux résidents de retourner dans leur maison pour Noël. Entre le Nouvel An et fin mars 1988, d'autres travaux se sont avérés nécessaires pour enlever une contamination résiduelle mineure préalablement masquée par la présence d'un niveau élevé de contamination.

L'opération a exigé, aux moments les plus critiques, quelque 250 professionnels et techniciens et quelque 300 personnes pour le support, le transport, la démolition, etc., à Goiânia même, ainsi que dans différents laboratoires à Rio de Janeiro.

\section{Information olu public et perception sociale}

Le soir de la découverte de l'accident, des rumeurs se sont répandues sur ce qui s'était passé. Elles ont été ensuite exacerbées le matin suivant lorsque les gens ont découvert, à leur réveil, des zones isolées sans qu'aucune explication cohérente ne leur soit donnée. De nombreuses personnes ont tenté de se rendre au stade olympique pour être rassurées, provoquant le surmenage des ressources humaines alors disponibles. L'intérêt du public et des médias s'est accru en augmentant l'épuisement des techniciens qui tentaient de maîtriser l'accident. Rètrospectivement, il est clair qu'il aurait fallu, dès le début, un attaché de presse, avec les appuis appropriés, pour informer le public.

On a déjà observé une réaction exagérée du public face aux accidents radiologiques en particulier après Three Mile Island et Tchernobyl, 
réaction qualifiée de "radiophobie". Elle a été observée après l'accident de Goiânia. Des personnes, par exemple, qui avaient été contaminées ou avaient été liées de quelque façon avec l'accident ont été traitées de "lépreux", même par leur propre famille. Le certificat de "non-contamination" délivré par le centre de contrôle installé au stade olympique était devenu presque indispensable pour se faire accepter par la communauté. Au total 112000 personnes ont été contrôlées. La "radiophobie" ne s'est pas limitée au public, elle a également gagné certains bureaux locaux et la profession médicale. L'exemple le plus frappant a été le lancement de pierres sur les cercueils lors de l'enterrement des quatre victimes. Cette "radiophobie" a dépassé les confins de Goiânia. Les ventes des principaux produits de l'État : bétail, céréales et autres produits agricoles, vêtements, produits à base de coton, ont baissé d'un quart durant la période qui a suivi l'accident.

Pour dissiper les craintes, on a incité les équipes chargées de la décontamination à expliquer à la population ce qu'elles faisaient et pourquoi. On leur a demandé notamment d'accepter l'eau et les aliments provenant des habitations. C'est ainsi qu'elles ont gagné la confiance de la population et accru la crédibilité des déclarations officielles. Cela a, cependant, également accru les besoins en ressources humaines. On a souvent montré à la télévision les équipes de décontamination ; et des causeries sur la radioprotection ont permis aux journalistes de mieux comprendre la situation. Une brochure intitulée " $\mathrm{Ce}$ que vous devez savoir sur la radioactivité et les rayonnements" a été distribuée à 250000 exemplaires. Un service téléphonique fonctionnant 24 heures sur 24 a été mis en place pour répondre aux questions.

\section{LES LEÇONS}

L'étude des faits a posteriori nourrit rétroactivement l'expérience en matière de systèmes de contrôle et d'intervention en cas d'accident. Elle permet d'une part de tirer des leçons et d'autre part d'illustrer les principes déjà connus et d'en souligner l'importance. Nous avons déjà vu ci-dessus quelques-uns des principaux points qui ont été dégagés de l'accident de Goiânia. Nous allons les résumer en y en ajoutant d'autres d'ordre plus général.

\section{Prévention et atténuation des conséquences}

1) L'accident, comme c'est souvent le cas, est la conséquence d'une abdication de responsabilité quant à la sécurité de la source. La vaste diffusion des événements qui se sont déroulés à Goiânia peut largement contribuer à encourager une attitude responsable dans la gestion des sources de rayonnement.

2) Il est évident que la forme aisément dispersable de la substance radioactive a aggravé l'accident. D'où l'idée qu'il faut utiliser, pour les sources scellées, chaque fois que c'est possible, des substances radioactives sous une forme chimique et physique qui ne soit pas facilement dispersable. 
3) Les deux hommes qui ont initialement démantelé l'unité de téléthérapie présentaient tous les symptômes d'une irradiation aiguë dès le début de l'accident. Si un diagnostic correct avait pu être posé pour eux ainsi que pour les personnes impliquées ultérieurement, l'ampleur de l'accident aurait pu être bien moindre. La difficulté de diagnostiquer les effets rarement rencontrés de l'exposition aux rayonnements est caractéristique de ce genre d'accidents. II n'y a pas si longtemps, la formation médicale prévoyait le diagnostic de piqûres d'insectes et d'araignées, de morsures de serpents, d'infections virales et d'exposition à des produits chimiques toxiques. L'AIEA est consciente de ce problème $[4,5]$. La diffusion de ses publications dans le milieu médical et le récit des événements de l'accident de Goiânia seront d'une grande utilité.

\section{Réaction initiale}

4) Pour faire face aux accidents radiologiques affectant le public, il devrait $y$ avoir un système bien connu permettant de demander des secours (de préférence au niveau local) et d'informer les autorités compétentes.

5) Comme dans toute catastrophe majeure, par exemple inondation, explosion, etc., les plans d'intervention des autorités locales doivent fournir un support précieux aux spécialistes en charge de ces urgences.

6) Dans ce genre d'accidents, la tendance générale, inspirée de considérations politiques et sociales, consiste à imposer des critères très restrictifs pour la mise en œuvre des contre-mesures. Ces actions sont économiquement assez lourdes et peuvent introduire des risques classiques, d'où l'importance de prévoir à l'avance des normes claires à utiliser en cas d'accident, basées sur l'étude approfondie de tous les problèmes impliqués.

7) Pour éviter le surmenage des hommes chargés de remédier aux conséquences de l'accident, tout en limitant l'inquiétude du public, les plans d'intervention doivent prévoir des dispositions adéquates pour répondre à l'exigence d'information du public et des médias.

\section{Aspect médical}

8) L'accident a démontré l'importance de pouvoir s'appuyer sur :

- des médecins ayant reçu une formation spécifique pour traiter les irradiés ;

- des accords internationaux permettant d'obtenir le secours de spécialistes.

9) C'est la première fois que l'on utilise aussi largement le bleu de Prusse pour accélérer la décorporation du césium. Les résultats obtenus au niveau métabolique ont été excellents, prouvant ainsi l'efficacité du traitement, même s'il a fallu augmenter les doses prescrites jusqu'alors. 
10) En cas d'accident radiologique, un équipement facilement transportable d'anthroporadiamétrie et d'analyses biologiques peut s'avérer nécessaire, en plus des installations permanentes.

11) Un seul hôpital centralisé, prévu pour traiter les victimes d'irradiations graves, peut ne pas être suffisant dans le cas d'un accident de l'ampleur de celui de Goiânia. II peut s'avérer nécessaire, pour les cas moins graves, de prévoir des dispositions permettant de les traiter localement.

\section{Opérations de réhabilitation}

12) Dans un accident de cette ampleur, on doit pouvoir disposer d'une équipe de professionnels qualifiés provenant de différents organismes, afin de définir clairement une structure de commandement et d'établir un support logistique intégrant toutes ces ressources. Des plans d'intervention devraient prévoir ces différents éléments.

13) L'accident de Goiânia, comme celui de Juares, a produit d'importants volumes de déchets radioactifs. La gestion des déchets est au cœur du programme de réhabilitation et n'est pas sans répercussions sur ce calendrier. II convient donc de prévoir des plans d'intervention appropriés pour traiter les gros volumes de déchets.

\section{CONCLUSIONS}

Les autorités brésiliennes ont bien su maîtriser l'accident de Goiânia, même si, comme elles l'ont déclaré dans le rapport de l'AIEA, certains aspects auraient pu être mieux traités. On peut se féliciter de leur franchise et leur volonté de collaborer avec l'AlEA pour rédiger un rapport des événements a posteriori, comme l'avait fait l'URSS après Tchernobyl. II est souhaitable qu'à l'avenir d'autres pays les imitent pour que nous puissions tous tirer des leçons de nos erreurs.

Un effort considérable a été fait pour l'amélioration des programmes d'intervention en cas d'accident nucléaire. Cet effort a été intensifié tant au niveau national qu'international après l'accident de Tchernobyl. En revanche, d'autres types d'accident radiologique n'ont pas bénéficié de la même attention. L'accident de Goiânia a, cependant, démontré l'ampleur potentielle des conséquences en échelle dans ce genre d'accident. Le rapport de l'AIEA souligne que les plans d'intervention devraient être étendus de façon à couvrir toute la gamme des accidents radiologiques possibles. Cela peut et doit être fait, mais il faut également veiller à ne pas déformer les faits. Nous devrions peut-être nous demander comment un tel accident, l'un des accidents radiologiques les plus graves, peut se comparer en termes de fréquence, de conséquences et de coût de prévention, à des accidents survenus dans d'autres domaines. Pour que la société puisse formuler des jugements bien fondés sur les priorités, il faut une grande compréhension des facteurs pertinents. Par le passé, le monde de la radioprotection ne s'est peut-être pas suffisamment tourné vers l'extérieur, ce qui n'a fait que permettre le développement d'une mystique sur ce sujet et masquer les véritables questions. 


\section{Remerciements}

Communication présentée lors du colloque des Communautés européennes "Second meeting of the Standing conference on Health and safety in the nuclear age: informing the public on improvements in emergency preparedness and nuclear accident management, Bruxelles, 5-6 décembre 1989. L'auteur remercie vivement Radioprotection pour la traduction de cette communication.

\section{RÉFÉRENCES}

[1] AGENCE INTERNATIONALE DE L'ÉNERGIE ATOMIQUE (AIEA) - Nuclear safety review for 1987. Vienne : AIEA, 1988.

[2] AGENCE INTERNATIONALE DE L'ÉNERGIE ATOMIQUE (AIEA) - The radiological accident in San Salvador. Vienne: AIEA, 1989.

[3] AGENCE INTERNATIONALE DE L'ÉNERGIE ATOMIQUE (AIEA) - L'accident radiologique de Goiânia. Vienne : AIEA, 1988.

[4] AGENCE INTERNATIONALE DE L'ÉNERGIE ATOMIQUE (AIEA) - What the general practitioner (MD) should know about medical handling of overexposed individuals (IAEA-TECDOC-366). Vienne : AIEA, 1986.

[5] AGENCE INTERNATIONALE DE L'ÉNERGIE ATOMIQUE (AIEA) - Medical handling of accidentally exposed individuals (Safety series $N^{\circ} 88$ ). Vienne : AIEA, 1988.

[6] COMISION NACIONAL DE SEGURIDAD NUCLEAR Y SALVAGUARDIAS - ACCidente por contamination con cobalto-60, Mexico 1984. Rapport CNSNSIT-001, Mexico, 1985.

[7] DE OLIVEIRA A.R. - Un répertoire des accidents radiologiques 1945-1985. Radioprotection, 1987, 22 (2), 89-135. 